\title{
New Class of duality models in discrete minmax fractional programming based on second-order univexities
}

\author{
Ram U. Verma ${ }^{1, *}$ and Tadeusz Antczak ${ }^{2}$ \\ ${ }^{1}$ Mathematical Sciences Division, International Publications, USA \\ ${ }^{2}$ Faculty of Mathematics and Computer Science, University of Lodz, Poland \\ (Received: 4 March 2017; Accepted: 15 August 2017)
}

\begin{abstract}
The main purpose of this paper is first to formulate four new general higher-order parametric duality models for a discrete minmax fractional programming problem, and then prove appropriate duality theorems utilizing various new classes of second-order $(\mathcal{F}, \beta, \phi, \zeta, \rho, \theta, m)$-univex functions.
\end{abstract}

Key Words Discrete minmax fractional programming; second-order $(\mathcal{F}, \beta, \phi, \zeta, \rho, \theta, m)$-univex functions; Generalized second-order parametric duality models; Duality theorems.

AMS Mathematics Subject Classification (2000): 90C26, 90C30, 90C32, 90C46, 90C47

DOI: $10.19139 /$ soic.v5i3.283

\section{Introduction and Preliminaries}

In this paper, we introduce four generalized second-order parametric duality models and prove a variety of weak, strong, and strict converse duality theorems using the notion of second-order $(\mathcal{F}, \beta, \phi, \zeta, \rho, \theta, m)$-univex functions for the following discrete minmax fractional programming problem:

(P) Minimize $\max _{1 \leq i \leq p} \frac{f_{i}(x)}{g_{i}(x)}$

subject to $G_{j}(x) \leq 0, \quad j \in \underline{q}, \quad H_{k}(x)=0, \quad k \in \underline{r}, \quad x \in X$,

where $X$ is an open convex subset of $\mathbb{R}^{n}$ (n-dimensional Euclidean space), $f_{i}, g_{i}, i \in p \equiv\{1,2, \ldots, p\}$, $G_{j}, j \in \underline{q}$, and $H_{k}, k \in \underline{r}$, are real-valued functions defined on $X$, and for each $i \in p, g_{i}(\bar{x})>0$ for all $x$ satisfying the constraints of $(P)$.

The problems of this nature are more frequently referred to as "generalized fractional programming problems" to the context of mathematical programming. These problems provide realistic models for some significant real-world problems (more notably encountered in multiobjective programming, approximation theory, goal programming, location planning and economics), while their mathematical tractability empowers equivalent parametric nonlinear programming problems with nonfractional objective functions.

Recently, Verma and Zalmai [10] established some second-order parametric necessary optimality conditions as well as numerous second-order sufficient optimality conditions for a discrete minmax fractional programming

*Correspondence to: Ram Verma (Email: verma99@msn.com)

ISSN 2310-5070 (online) ISSN 2311-004X (print)

Copyright (C) 2017 International Academic Press 
problem applying various second-order $(\phi, \eta, \rho, \theta, m)$-invexity assumptions. This second-order invexity generalizes the concepts of invexity, pseudoinvexity, and quasiinvexity, originally defined by Hanson [3]. The second-order invexity is also referred to as "sonvexity" in the literature.

In the present paper, we plan to introduce new classes of second-order $(\mathcal{F}, \beta, \phi, \zeta, \rho, \theta, m)$-univex functions, which generalize most of the existing notions of second-order univex functions. The second-order univex functions are also referred to as "sounivex functions" in the literature. Here, we shall utilize two partitioning schemes due to Mond and Weir [6] and Yang [13], in conjunction with the generalized versions of the new classes of second-order $(\mathcal{F}, \beta, \phi, \zeta, \rho, \theta, m)$-univex functions to formulate four generalized duality models for $(P)$ and prove appropriate duality theorems. The duality models and the related duality theorems established in this paper generalize those presented in [10 - 12] and others. To the best of our knowledge, all of these duality results are new in the area of discrete minmax fractional programming. In fact, it seems that results of this type, which are based on second-order necessary and sufficient optimality conditions, have not yet appeared in any shape or form for any type of mathematical programming problems. For extensive lists of publications dealing with second-order and higher-order duality results for various categories of nonlinear programming problems, which are essentially based on first-order optimality conditions and different kinds of generalized convexity concepts, we refer the reader ([2]-[5], [7], [9]-[12], [15], [16]).

All the parametric duality results established in this paper can easily be modified (and specialized) for each one of the following three classes of nonlinear programming problems to the context $(P)$ :

$$
\begin{array}{ll}
\underset{x \in \mathbb{F}}{\operatorname{Minimize}} & \frac{f_{1}(x)}{g_{1}(x)} ; \\
\underset{x \in \mathbb{F}}{\operatorname{Minimize}} & \max _{1 \leq i \leq p} f_{i}(x) ; \\
\underset{x \in \mathbb{F}}{\operatorname{Minimize}} & f_{1}(x),
\end{array}
$$

where $\mathbb{F}$ (assumed to be nonempty) is the feasible set of $(P)$, that is,

$$
\mathbb{F}=\left\{x \in X: G_{j}(x) \leq 0, \quad j \in \underline{q}, \quad H_{k}(x)=0, \quad k \in \underline{r}\right\} .
$$

The paper is organized as follows: In Section 1, we introduce a few basic definitions and recall some auxiliary results that will be used in the sequel. In Section 2, we utilize a partitioning scheme due to Mond and Weir [6], and formulate two general second-order parametric duality models for $(P)$ and prove weak, strong, and strict converse duality theorems using various generalized $(\mathcal{F}, \beta, \phi, \zeta, \rho, \theta, m)$-sounivexity assumptions. In Section 3 , we shall make use of another partitioning method due to Yang [13] and construct another pair of general second-order parametric duality models with different constraint structures and discuss several second-order duality results under a variety of generalized $(\mathcal{F}, \beta, \phi, \zeta, \rho, \theta, m)$-sounivexity assumptions. Finally, in Section 4 we present some remarks on our main results aiming at some future research endeavors arising from certain modifications of the principal minmax model formulated in the present investigation.

We next introduce some generalized versions of the sounivexity (Zalmai [15]) and others in the literature. Let $f: X \rightarrow \mathbb{R}$ be a twice differentiable function. Suppose that $\|\cdot\|$ denotes a norm on $\mathbb{R}^{n}$, and $\langle a, b\rangle$ is the inner product of the vectors $a$ and $b$.

Definition 1.1. The function $f$ is said to be (strictly) $(\mathcal{F}, \beta, \phi, \rho, \zeta, \theta, m)$-sounivex at $x^{*} \in X$ if there exist functions $\beta: X \times X \rightarrow \mathbb{R}_{+} \backslash\{0\}, \phi: \mathbb{R} \rightarrow \mathbb{R}, \rho: X \times X \rightarrow \mathbb{R}, \theta, \zeta: X \times X \rightarrow \mathbb{R}^{n}$, a sublinear function $\mathcal{F}\left(x, x^{*} ; \cdot\right): \mathbb{R}^{n} \rightarrow \mathbb{R}$, and a positive integer $m$ such that for each $x \in X\left(x \neq x^{*}\right)$ and $z \in \mathbb{R}^{n}$,

$$
\phi\left(f(x)-f\left(x^{*}\right)\right)(>) \geq \mathcal{F}\left(x, x^{*} ; \beta\left(x, x^{*}\right) \nabla f\left(x^{*}\right)\right)+\frac{1}{2}\left\langle\zeta\left(x, x^{*}\right), \nabla^{2} f\left(x^{*}\right) z\right\rangle+\rho\left(x, x^{*}\right)\left\|\theta\left(x, x^{*}\right)\right\|^{m} .
$$


The function $f$ is said to be (strictly) $(\mathcal{F}, \beta, \phi, \rho, \zeta, \theta, m)$-sounivex on $X$ if it is (strictly) $(\mathcal{F}, \beta, \phi, \rho, \theta, m)$ sounivex at each $x^{*} \in X$.

Definition 1.2. The function $f$ is said to be (strictly) $(\mathcal{F}, \beta, \phi, \rho, \theta, m)$-pseudosounivex at $x^{*} \in X$ if there exist functions $\beta: X \times X \rightarrow \mathbb{R}_{+} \backslash\{0\}, \phi: \mathbb{R} \rightarrow \mathbb{R}, \rho: X \times X \rightarrow \mathbb{R}, \theta, \zeta: X \times X \rightarrow \mathbb{R}^{n}$, a sublinear function $\mathcal{F}\left(x, x^{*} ; \cdot\right): \mathbb{R}^{n} \rightarrow \mathbb{R}$, and a positive integer $m$ such that for each $x \in X\left(x \neq x^{*}\right)$ and $z \in \mathbb{R}^{n}$,

$$
\begin{aligned}
\mathcal{F}\left(x, x^{*} ; \beta\left(x, x^{*}\right) \nabla f\left(x^{*}\right)\right)+\frac{1}{2}\left\langle\zeta\left(x, x^{*}\right), \nabla^{2} f\left(x^{*}\right) z\right\rangle \geq-\rho\left(x, x^{*}\right)\left\|\theta\left(x, x^{*}\right)\right\|^{m} & \\
& \Rightarrow \phi\left(f(x)-f\left(x^{*}\right)\right)(>) \geq 0 .
\end{aligned}
$$

The function $f$ is said to be (strictly) $(\mathcal{F}, \beta, \phi, \rho, \zeta, \theta, m)$-pseudosounivex on $X$ if it is (strictly) $(\mathcal{F}, \beta, \phi, \rho, \zeta, \theta, m)$-pseudosounivex at each $x^{*} \in X$.

Definition 1.3. The function $f$ is said to be (prestrictly) $(\mathcal{F}, \beta, \phi, \rho, \zeta, \theta, m)$-quasisounivex at $x^{*} \in X$ if there exist functions $\beta: X \times X \rightarrow \mathbb{R}_{+} \backslash\{0\}, \phi: \mathbb{R} \rightarrow \mathbb{R}, \rho: X \times X \rightarrow \mathbb{R}, \theta, \zeta: X \times X \rightarrow \mathbb{R}^{n}$, a sublinear function $\mathcal{F}\left(x, x^{*} ; \cdot\right): \mathbb{R}^{n} \rightarrow \mathbb{R}$, and a positive integer $m$ such that for each $x \in X$ and $z \in \mathbb{R}^{n}$,

$$
\begin{aligned}
\phi\left(f(x)-f\left(x^{*}\right)\right)(<) \leq 0 & \\
& \Rightarrow \mathcal{F}\left(x, x^{*} ; \beta\left(x, x^{*}\right) \nabla f\left(x^{*}\right)\right)+\frac{1}{2}\left\langle\zeta\left(x, x^{*}\right), \nabla^{2} f\left(x^{*}\right) z\right\rangle \leq-\rho\left(x, x^{*}\right)\left\|\theta\left(x, x^{*}\right)\right\|^{m} .
\end{aligned}
$$

The function $f$ is said to be (prestrictly) $(\mathcal{F}, \beta, \phi, \rho, \zeta, \theta, m)$-quasisounivex on $X$ if it is (prestrictly) $(\mathcal{F}, \beta, \phi, \rho, \zeta, \theta, m)$-quasisounivex at each $x^{*} \in X$.

From the above definitions it is clear that if $f$ is $(\mathcal{F}, \beta, \phi, \rho, \zeta, \theta, m)$-sounivex at $x^{*}$, then it is both $(\mathcal{F}, \beta, \phi, \rho, \zeta, \theta, m)$-pseudosounivex and $(\mathcal{F}, \beta, \phi, \rho, \zeta, \theta, m)$-quasisounivex at $x^{*} \in X$, if $f$ is $(\mathcal{F}, \beta, \phi, \rho, \theta, m)$ quasisounivex at $x^{*} \in X$, then it is prestrictly $(\mathcal{F}, \beta, \phi, \rho, \theta, m)$-quasisounivex at $x^{*} \in X$, and if $f$ is strictly $(\mathcal{F}, \beta, \phi, \rho, \zeta, \theta, m)$-pseudosounivex at $x^{*} \in X$, then it is $(\mathcal{F}, \beta, \phi, \rho, \zeta, \theta, m)$-quasisounivex at $x^{*} \in X$.

We observe that in some contexts during proving the duality theorems, it may be easier to apply certain alternative but equivalent forms of the above definitions by considering the contrapositive statements. For example, $(\mathcal{F}, \beta, \phi, \rho, \zeta, \theta, m)$-quasisounivexity can be defined in the following equivalent way:

The function $f$ is said to be $(\mathcal{F}, \beta, \phi, \rho, \zeta, \theta, m)$-quasisounivex at $x^{*} \in X$ if there exist functions $\beta: X \times$ $X \rightarrow \mathbb{R}_{+} \backslash\{0\}, \phi: \mathbb{R} \rightarrow \mathbb{R}, \rho: X \times X \rightarrow \mathbb{R}, \theta, \zeta: X \times X \rightarrow \mathbb{R}^{n}$, a sublinear function $\left(x, x^{*} ; \cdot\right): \mathbb{R}^{n} \rightarrow \mathbb{R}$, and a positive integer $m$ such that for each $x \in X$ and $z \in \mathbb{R}^{n}$,

$$
\mathcal{F}\left(x, x^{*} ; \beta\left(x, x^{*}\right) \nabla f\left(x^{*}\right)\right)+\frac{1}{2}\left\langle\zeta\left(x, x^{*}\right), \nabla^{2} f\left(x^{*}\right) z\right\rangle>-\rho\left(x, x^{*}\right)\left\|\theta\left(x, x^{*}\right)\right\|^{m}
$$

$$
\Rightarrow \phi\left(f(x)-f\left(x^{*}\right)\right)>0 .
$$

As the generalized sounivexity of functions generalizes most of the convex functions, including generalized invexity, pseudoinvexity and quasiinvexity by identifying appropriate choices of $\mathcal{F}, \beta, \phi, \rho, \zeta, \theta$, and $m$. For example, if let $\mathcal{F}\left(x, x^{*} ; \nabla f\left(x^{*}\right)\right)=\left\langle\nabla f\left(x^{*}\right), \eta\left(x, x^{*}\right)\right\rangle$ and $\beta\left(x, x^{*}\right) \equiv 1$, where $\eta$ is a given function from $X \times X$ to $\mathbb{R}^{n}$, then we obtain the definitions of $(\phi, \eta, \rho, \zeta, \theta, m)$-sonvex, $(\phi, \eta, \rho, \zeta, \theta, m)$-pseudosonvex, and $(\phi, \eta, \rho, \zeta, \theta, m)$-quasisonvex functions introduced recently in [10]. For example, let $f: X \rightarrow \mathbb{R}$ be a twice differentiable function.

Definition 1.4. The function $f$ is said to be (strictly) $(\phi, \eta, \zeta, \rho, \theta, m)$-sonvex at $x^{*}$ if there exist functions $\phi: \mathbb{R} \rightarrow \mathbb{R}, \eta, \zeta: X \times X \rightarrow \mathbb{R}^{n}, \rho: X \times X \rightarrow \mathbb{R}$, and $\theta: X \times X \rightarrow \mathbb{R}^{n}$, and a positive integer $m$ such that for each $x \in X\left(x \neq x^{*}\right)$ and $z \in \mathbb{R}^{n}$,

$$
\phi\left(f(x)-f\left(x^{*}\right)\right)(>) \geq\left\langle\nabla f\left(x^{*}\right), \eta\left(x, x^{*}\right)\right\rangle+\frac{1}{2}\left\langle\zeta\left(x, x^{*}\right), \nabla^{2} f\left(x^{*}\right) z\right\rangle+\rho\left(x, x^{*}\right)\left\|\theta\left(x, x^{*}\right)\right\|^{m} .
$$


Definition 1.5. The function $f$ is said to be (strictly) $(\phi, \eta, \rho, \theta, m)$-sonvex at $x^{*}$ if there exist functions $\phi$ : $\mathbb{R} \rightarrow \mathbb{R}, \eta: X \times X \rightarrow \mathbb{R}^{n}, \rho: X \times X \rightarrow \mathbb{R}$, and $\theta: X \times X \rightarrow \mathbb{R}^{n}$, and a positive integer $m$ such that for each $x \in X\left(x \neq x^{*}\right)$ and $z \in \mathbb{R}^{n}$,

$$
\phi\left(f(x)-f\left(x^{*}\right)\right)(>) \geq\left\langle\nabla f\left(x^{*}\right), \eta\left(x, x^{*}\right)\right\rangle+\frac{1}{2}\left\langle z, \nabla^{2} f\left(x^{*}\right) z\right\rangle+\rho\left(x, x^{*}\right)\left\|\theta\left(x, x^{*}\right)\right\|^{m} .
$$

We next present an example on some generalized versions of the sounivexity (Zalmai [15]) and others in the literature.

Example 1. Let $f: X \rightarrow \mathbb{R}$ be a twice differentiable function with the norm $\|\cdot\|$ on $\mathbb{R}^{n}$ and inner product $\langle a, b\rangle$ of the vectors $a$ and $b$. Then the function $f$ is said to be (strictly) $(\mathcal{F}, \beta, \phi, \rho, \xi, \theta, m)$-sounivex at $x^{*}$ if there exist functions $\beta: X \times X \rightarrow \mathbb{R}_{+} \backslash\{0\}, \phi: \mathbb{R} \rightarrow \mathbb{R}, \rho: X \times X \rightarrow \mathbb{R}, \theta, \xi: X \times X \rightarrow \mathbb{R}^{n}$, a sublinear function $\mathcal{F}\left(x, x^{*} ; \cdot\right): \mathbb{R}^{n} \rightarrow \mathbb{R}$, and a positive integer $m$ such that for each $x \in X\left(x \neq x^{*}\right)$ and $z \in \mathbb{R}^{n}$,

$$
\begin{aligned}
& \phi\left(f(x)-f\left(x^{*}\right)+\frac{1}{2}\left\langle z, \nabla^{2} f\left(x^{*}\right) z\right\rangle\right)(>) \geq \mathcal{F}\left(x, x^{*} ; \beta\left(x, x^{*}\right) \nabla f\left(x^{*}\right)\right)+\left\langle\xi\left(x, x^{*}\right), \nabla^{2} f\left(x^{*}\right) z\right\rangle \\
& +\rho\left(x, x^{*}\right)\left\|\theta\left(x, x^{*}\right)\right\|^{m} .
\end{aligned}
$$

We conclude this section by recalling a set of second-order necessary optimality conditions for $(P)$.

Theorem 1.1. [15] Let $x^{*}$ be an optimal solution of $(P)$, let $\lambda^{*}=\varphi\left(x^{*}\right) \equiv$ $\left.\max _{1 \leq i \leq p} f_{i}\left(x^{*}\right) / g_{i}\left(x^{*}\right)\right\}$, and assume that the functions $f_{i}, g_{i}, i \in \underline{p}, G_{j}, j \in \underline{q}$, and $H_{k}, k \in \underline{r}$, are twice continuously differentiable at $x^{*}$, and that the second-order Guignard constraint qualification holds at $x^{*}$. Then for each $z^{*} \in C\left(x^{*}\right)$, there exist $u^{*} \in U \equiv\left\{u \in \mathbb{R}^{p}: u \geq 0, \sum_{i=1}^{p} u_{i}=1\right\}, v^{*} \in \mathbb{R}_{+}^{q} \equiv\left\{v \in \mathbb{R}^{q}: v \geq 0\right\}$, and $w^{*} \in \mathbb{R}^{r}$ such that

$$
\begin{gathered}
\sum_{i=1}^{p} u_{i}^{*}\left[\nabla f_{i}\left(x^{*}\right)-\lambda^{*} \nabla g_{i}\left(x^{*}\right)\right]+\sum_{j=1}^{q} v_{j}^{*} \nabla G_{j}\left(x^{*}\right)+\sum_{k=1}^{r} w_{k}^{*} \nabla H_{k}\left(x^{*}\right)=0, \\
\left\langle z^{*},\left\{\sum_{i=1}^{p} u_{i}^{*}\left[\nabla^{2} f_{i}\left(x^{*}\right)-\lambda^{*} \nabla^{2} g_{i}\left(x^{*}\right)\right]+\sum_{j=1}^{q} v_{j}^{*} \nabla^{2} G_{j}\left(x^{*}\right)+\sum_{k=1}^{r} w_{k}^{*} \nabla^{2} H_{k}\left(x^{*}\right)\right\} z^{*}\right\rangle \geq 0, \\
u_{i}^{*}\left[f_{i}\left(x^{*}\right)-\lambda^{*} g_{i}\left(x^{*}\right)\right]=0, \quad i \in \underline{p}, \\
v_{j}^{*} G_{j}\left(x^{*}\right)=0, \quad j \in \underline{q},
\end{gathered}
$$

where $C\left(x^{*}\right)$ is the set of all critical directions of $(P)$ at $x^{*}$, that is,

$$
\begin{aligned}
& C\left(x^{*}\right)=\left\{z \in \mathbb{R}^{n}:\left\langle\nabla f_{i}\left(x^{*}\right)-\lambda \nabla g_{i}\left(x^{*}\right), z\right\rangle=0, \quad i \in A\left(x^{*}\right), \quad\left\langle\nabla G_{j}\left(x^{*}\right), z\right\rangle \leq 0, \quad j \in B\left(x^{*}\right),\right. \\
& \left.\left\langle\nabla H_{k}\left(x^{*}\right), z\right\rangle=0, \quad k \in \underline{r}\right\} \\
& A\left(x^{*}\right)=\left\{j \in \underline{p}: f_{j}\left(x^{*}\right) / g_{j}\left(x^{*}\right)=\max _{1 \leq i \leq p} f_{i}\left(x^{*}\right) / g_{i}\left(x^{*}\right)\right\}, \text { and } B\left(x^{*}\right)=\left\{j \in \underline{q}: G_{j}\left(x^{*}\right)=0\right\} \text {. }
\end{aligned}
$$

For brevity, we shall henceforth refer to $x^{*}$ as a normal optimal solution of $(P)$ if it is an optimal solution and satisfies the second-order Guignard constraint qualification.

In the remainder of this paper, we shall assume that the functions $f_{i}, g_{i}, i \in \underline{p}, G_{j}, j \in \underline{q}$, and $H_{k}, k \in \underline{r}$, are twice continuously differentiable on the open set $X$. Moreover, we shall assume, without loss of generality, that $g_{i}(x)>0, i \in \underline{p}$, and $\varphi(x) \geq 0$ for all $x \in X$. 


\section{Duality Model I}

In this section, we discuss several families of duality results under various generalized $(\mathcal{F}, \beta, \phi, \rho, \zeta, \theta, m)$ sounivexity hypotheses imposed on certain combinations of the functions involved in the considered optimization problem. This is accomplished by employing a certain partitioning scheme which was originally proposed in [6] for the purpose of constructing generalized dual problems for nonlinear programming problems. For this we need some additional notation.

Let $\left\{J_{0}, J_{1}, \ldots, J_{M}\right\}$ and $\left\{K_{0}, K_{1}, \ldots, K_{M}\right\}$ be partitions of the index sets $\underline{q}$ and $\underline{r}$, respectively; thus, $J_{\mu} \subseteq \underline{q}$ for each $\mu \in \underline{M} \cup\{0\}, J_{\mu} \cap J_{\nu}=\emptyset$ for each $\mu, \nu \in \underline{m} \cup\{0\}$ with $\mu \neq \nu$, and $\cup_{\mu=0}^{m} J_{\mu}=q$. Obviously, similar properties hold for $\left\{K_{0}, K_{1}, \ldots, K_{M}\right\}$. Moreover, if $M_{1}$ and $M_{2}$ are the numbers of the partitioning sets of $q$ and $\underline{r}$, respectively, then $M=\max \left\{M_{1}, M_{2}\right\}$ and $J_{\mu}=\emptyset$ or $K_{\mu}=\emptyset$ for $\mu>\min \left\{M_{1}, M_{2}\right\}$.

In addition, we use the real-valued functions $\xi \rightarrow \Phi_{i}(\xi, v, w, \lambda), i \in \underline{p}, \xi \rightarrow \Phi(\xi, u, v, w, \lambda)$, and $\xi \rightarrow$ $\Lambda_{t}(\xi, v, w)$ defined, for fixed $\lambda, u, v$, and $w$, on $X$ as follows:

$$
\begin{gathered}
\Phi_{i}(\xi, v, w, \lambda)=f_{i}(\xi)-\lambda g_{i}(\xi)+\sum_{j \in J_{0}} v_{j} G_{j}(\xi)+\sum_{k \in K_{0}} w_{k} H_{k}(\xi), \quad i \in \underline{p}, \\
\Phi(\xi, u, v, w, \lambda)=\sum_{i=1}^{p} u_{i}\left[f_{i}(\xi)-\lambda g_{i}(\xi)\right]+\sum_{j \in J_{0}} v_{j} G_{j}(\xi)+\sum_{k \in K_{0}} w_{k} H_{k}(\xi), \\
\Lambda_{t}(\xi, v, w,)=\sum_{j \in J_{t}} v_{j} G_{j}(\xi)+\sum_{k \in K_{t}} w_{k} H_{k}(\xi), \quad t \in \underline{M} .
\end{gathered}
$$

Making use of the sets and functions defined above, we can now formulate our first pair of second-order parametric duality models for $(P)$.

Consider the following two problems:

$(D I) \quad$ Maximize $\lambda$

subject to

$$
\begin{gathered}
\sum_{i=1}^{p} u_{i}\left[\nabla f_{i}(y)-\lambda \nabla g_{i}(y)\right]+\sum_{j=1}^{q} v_{j} \nabla G_{j}(y)+\sum_{k=1}^{r} w_{k} \nabla H_{k}(y)=0 \\
\left\langle z,\left\{\sum_{i=1}^{p} u_{i}\left[\nabla^{2} f_{i}(y)-\lambda \nabla^{2} g_{i}(y)\right]+\sum_{j=1}^{q} v_{j} \nabla^{2} G_{j}(y)+\sum_{k=1}^{r} w_{k} \nabla^{2} H_{k}(y)\right\} z\right\rangle \geq 0 \\
f_{i}(y)-\lambda g_{i}(y)+\sum_{j \in J_{0}} v_{j} G_{j}(y)+\sum_{k \in K_{0}} w_{k} H_{k}(y) \geq 0, \quad i \in \underline{p} \\
\sum_{j \in J_{t}} v_{j} G_{j}(y)+\sum_{k \in K_{t}} w_{k} H_{k}(y) \geq 0, \quad t \in \underline{M}, \\
y \in X, z \in C(y), u \in \mathbb{R}^{p}, u \geq 0, \sum_{i=1}^{p} u_{i}=1, v \in \mathbb{R}_{+}^{q}, w \in \mathbb{R}^{r}, \lambda \in \mathbb{R}_{+}
\end{gathered}
$$

$(\tilde{D} I) \quad$ Maximize $\quad \lambda$

subject to (2.2) - (2.5) and

$$
\mathcal{F}\left(x, y ; \sum_{i=1}^{p} u_{i}\left[\nabla f_{i}(y)-\lambda \nabla g_{i}(y)\right]+\sum_{j=1}^{q} v_{j} \nabla G_{j}(y)+\sum_{k=1}^{r} w_{k} \nabla H_{k}(y)\right) \geq 0 \text { for all } x \in \mathbb{F},
$$


where $\mathcal{F}(x, y ; \cdot)$ is a sublinear function from $\mathbb{R}^{n}$ to $\mathbb{R}$.

The feasible set $\mathbb{F}_{(D I)}$ (assumed to be nonempty) of $(D I)$ is defined as:

$$
\mathbb{F}_{(D I)}=\left\{y \in X: z \in \mathbb{R}^{n} ; u \in \mathbb{R}^{p}, u \geq 0, \sum_{i=1}^{p} u_{i}=1 ; v \in \mathbb{R}_{+}^{q}, v_{i} \geq 0 ; w \in \mathbb{R}^{r} ; \lambda \in \mathbb{R}_{+}\right\}
$$

Comparing $(D I)$ and $(\tilde{D} I)$, we see that $(\tilde{D} I)$ is relatively more general than $(D I)$ in the sense that any feasible solution of $(D I)$ is also feasible for $(\tilde{D} I)$, but the converse is not necessarily true. Furthermore, we observe that (2.1) is a system of $n$ equations, whereas (2.6) is a single inequality. Clearly, from a computational point of view, $(D I)$ is preferable to $(\tilde{D} I)$ because of the dependence of (2.6) on the feasible set of $(P)$.

Despite these apparent differences, it turns out that the statements and proofs of all the duality theorems for $(P)-(D I)$ and $(P)-(\tilde{D} I)$ are almost identical and, therefore, we shall consider only the pair $(P)-(D I)$.

In the proofs of our duality theorems, we shall make frequent use of the following auxiliary result which provides an alternative expression for the objective function of $(P)$.

Lemma 1. [10] For each $x \in X$,

$$
\varphi(x)=\max _{1 \leq i \leq p} \frac{f_{i}(x)}{g_{i}(x)}=\max _{u \in U} \frac{\sum_{i=1}^{p} u_{i} f_{i}(x)}{\sum_{i=1}^{p} u_{i} g_{i}(x)} .
$$

The next two theorems show that $(D I)$ is a dual problem for $(P)$.

Theorem 2.1. (Weak Duality) Let $x$ and y be arbitrary feasible solutions of $(P)$ and $(D I)$, respectively. Furthermore, assume that any one of the following four sets of hypotheses is satisfied:

(a) (i) $\xi \rightarrow \Phi(\xi, u, v, w, \lambda)$ is $(\mathcal{F}, \beta, \bar{\phi}, \bar{\rho}, \zeta, \theta, m)$-pseudosounivex at $y$ and $\bar{\phi}(a) \geq 0 \Rightarrow a \geq 0$;

(ii) for each $t \in \underline{M}, \xi \rightarrow \Lambda_{t}(\xi, v, w)$ is $\left(\mathcal{F}, \beta, \tilde{\phi}_{t}, \tilde{\rho}_{t}, \zeta, \theta, m\right)$-quasisounivex at $y$, $\tilde{\phi}_{t}$ is increasing, and $\tilde{\phi}_{t}(0)=0$

(iii) $\bar{\rho}(x, y)+\sum_{t=1}^{M} \tilde{\rho}_{t}(x, y) \geq 0$;

(b) (i) $\xi \rightarrow \Phi(\xi, u, v, w, \lambda)$ is prestrictly $(\mathcal{F}, \beta, \bar{\phi}, \bar{\rho}, \zeta, \theta, m)$-quasisounivex at $y$ and $\bar{\phi}(a) \geq 0 \Rightarrow a \geq 0$;

(ii) for each $t \in \underline{M}, \xi \rightarrow \Lambda_{t}(\xi, v, w)$ is $\left(\mathcal{F}, \beta, \tilde{\phi}_{t}, \rho_{t}, \zeta, \theta, m\right)$-quasisounivex at $y$, $\tilde{\phi}_{t}$ is increasing, and $\tilde{\phi}_{t}(0)=0$

(iii) $\bar{\rho}(x, y)+\sum_{t=1}^{M} \tilde{\rho}_{t}(x, y)>0$;

(c) (i) $\xi \rightarrow \Phi(\xi, u, v, w, \lambda)$ is prestrictly $(\mathcal{F}, \beta, \bar{\phi}, \bar{\rho}, \zeta, \theta, m)$-quasisounivex at $y$ and $\bar{\phi}(a) \geq 0 \Rightarrow a \geq 0$;

(ii) for each $t \in \underline{M}, \xi \rightarrow \Lambda_{t}(\xi, v, w)$ is strictly $\left(\mathcal{F}, \beta, \tilde{\phi}_{t}, \tilde{\rho}_{t}, \zeta, \theta, m\right)$-pseudosounivex at $y$, $\tilde{\phi}_{t}$ is increasing, and $\tilde{\phi}_{t}(0)=0$

(iii) $\bar{\rho}(x, y)+\sum_{t=1}^{M} \tilde{\rho}_{t}(x, y) \geq 0$;

(d) (i) $\xi \rightarrow \Phi(\xi, u, v, w, \lambda)$ is prestrictly $(\mathcal{F}, \beta, \bar{\phi}, \bar{\rho}, \zeta, \theta, m)$-quasisounivex at $y$ and $\bar{\phi}(a) \geq 0 \Rightarrow a \geq 0$;

(ii) for each $t \in \underline{M_{1}}, \xi \rightarrow \Lambda_{t}(\xi, v, w)$ is $\left(\mathcal{F}, \beta, \tilde{\phi}_{t}, \tilde{\rho}_{t}, \zeta, \theta, m\right)$-quasisounivex at $y$, for each $t \in \underline{M_{2}} \neq$ $\emptyset, \xi \rightarrow \Lambda_{t}(\xi, \overline{v, w})$ is strictly $\left(\mathcal{F}, \beta, \tilde{\phi}_{t}, \tilde{\rho}_{t}, \zeta, \theta, m\right)$-pseudosounivex at $y$, and for each $t \in \underline{M}, \overline{\tilde{\phi}_{t}}$ is increasing and $\tilde{\phi}_{t}(0)=0$, where $\left\{\underline{M_{1}}, \underline{M_{2}}\right\}$ is a partition of $\underline{M}$;

(iii) $\bar{\rho}(x, y)+\sum_{t=1}^{M} \tilde{\rho}_{t}(x, y) \geq 0$.

Then $\varphi(x) \geq \lambda$.

Proof

(a): Since $\mathcal{F}(x, y ; \cdot)$ is sublinear, it is clear that (2.1) and (2.2) can be expressed as follows: 


$$
\begin{gathered}
\mathcal{F}\left(x, y ; \beta(x, y)\left\{\sum_{i=1}^{p} u_{i}\left[\nabla f_{i}(y)-\lambda \nabla g_{i}(y)\right]+\sum_{j \in J_{0}} v_{j} \nabla G_{j}(y)+\right.\right. \\
\left.\left.\sum_{k \in K_{0}} w_{k} \nabla H_{k}(y)\right\}\right)+\mathcal{F}\left(x, y ; \beta(x, y) \sum_{t=1}^{M}\left[\sum_{j \in J_{t}} v_{j} \nabla G_{j}(y)+\sum_{k \in K_{t}} w_{k} \nabla H_{k}(y)\right]\right) \geq 0 . \\
\frac{1}{2}\left\langle\zeta(x, y),\left\{\sum_{i=1}^{p} u_{i}\left[\nabla^{2} f_{i}(y)-\lambda \nabla^{2} g_{i}(y)\right]+\sum_{j \in J_{0}} v_{j} \nabla^{2} G_{j}(y)+\right.\right. \\
\left.\left.\sum_{k \in K_{0}} w_{k} \nabla^{2} H_{k}(y)\right\} z\right\rangle+\frac{1}{2}\left\langle\zeta(x, y), \sum_{t=1}^{M}\left[\sum_{j \in J_{t}} v_{j} \nabla^{2} G_{j}(y)+\sum_{k \in K_{t}} w_{k} \nabla^{2} H_{k}(y)\right] z\right\rangle \geq 0 .
\end{gathered}
$$

Since for each $t \in \underline{M}$,

$$
\begin{aligned}
\Lambda_{t}(x, v, w) & =\sum_{j \in J_{t}} v_{j} G_{j}(x)+\sum_{k \in K_{t}} w_{k} H_{k}(x) \\
& \leq 0 \text { (by the primal feasibility of } x \text { ) } \\
& \leq \sum_{j \in J_{t}} v_{j} G_{j}(y)+\sum_{k \in K_{t}} w_{k} H_{k}(y)
\end{aligned}
$$

$$
\begin{aligned}
& \text { (by (2.4) and the dual feasibility of } y \text { ) } \\
= & \Lambda_{t}(y, v, w),
\end{aligned}
$$

it implies

$$
\tilde{\phi}_{t}\left(\Lambda_{t}(x, v, w)-\Lambda_{t}(y, v, w)\right) \leq 0
$$

Thus, it follows from (ii) that

$$
\begin{aligned}
\mathcal{F}\left(x, y ; \beta(x, y)\left[\sum_{j \in J_{t}} v_{j} \nabla G_{j}(y)+\sum_{k \in K_{t}} w_{k} \nabla H_{k}(y)\right]\right)+\frac{1}{2}\left\langle\zeta(x, y),\left[\sum_{j \in J_{t}} v_{j} \nabla^{2} G_{j}(y)\right.\right. & \\
& \left.\left.+\sum_{k \in K_{t}} w_{k} \nabla^{2} H_{k}(y)\right] z\right\rangle \leq-\tilde{\rho}_{t}(x, y)\|\theta(x, y)\|^{m} .
\end{aligned}
$$

Summing over $t \in \underline{M}$ and using the sublinearity of $\mathcal{F}(x, y ; \cdot)$, we obtain

$$
\begin{aligned}
\mathcal{F}\left(x, y ; \beta(x, y) \sum_{t=1}^{M}\left[\sum_{j \in J_{t}} v_{j} \nabla G_{j}(y)+\sum_{k \in K_{t}} w_{k} \nabla H_{k}(y)\right]\right)+\frac{1}{2}\left\langle\zeta(x, y), \sum_{t=1}^{M}\left[\sum_{j \in J_{t}} v_{j} \nabla^{2} G_{j}(y)\right.\right. \\
\left.\left.+\sum_{k \in K_{t}} w_{k} \nabla^{2} H_{k}(y)\right] z\right\rangle \leq-\sum_{t=1}^{M} \tilde{\rho}_{t}(x, y)\|\theta(x, y)\|^{m}
\end{aligned}
$$


Combining (2.7) - (2.9), and using (iii) we get

$$
\begin{aligned}
\mathcal{F}\left(x, y ; \beta(x, y)\left\{\sum_{i=1}^{p} u_{i}\left[\nabla f_{i}(y)-\lambda \nabla g_{i}(y)\right]+\sum_{j \in J_{0}} v_{j} \nabla G_{j}(y)+\sum_{k \in K_{0}} w_{k} \nabla H_{k}(y), \eta(x, y)\right\}\right) \\
+\frac{1}{2}\left\langle\zeta(x, y),\left\{\sum_{i=1}^{p} u_{i}\left[\nabla^{2} f_{i}(y)-\lambda \nabla^{2} g_{i}(y)\right]+\sum_{j \in J_{0}} v_{j} \nabla^{2} G_{j}(y)+\sum_{k \in K_{0}} w_{k} \nabla^{2} H_{k}(y)\right\} z\right\rangle \\
\geq \sum_{t=1}^{M} \tilde{\rho}_{t}(x, y)\|\theta(x, y)\|^{m} \geq-\bar{\rho}(x, y)\|\theta(x, y)\|^{m} .
\end{aligned}
$$

By (i), the above inequality implies that

$$
\bar{\phi}(\Phi(x, u, v, w, \lambda)-\Phi(y, u, v, w, \lambda)) \geq 0 .
$$

But $\bar{\phi}(a) \geq 0 \Rightarrow a \geq 0$, and hence we get

$$
\Phi(x, u, v, w, \lambda) \geq \Phi(y, u, v, w, \lambda) \geq 0,
$$

where the second inequality follows from (2.3) and the dual feasibility of $y$. Since $x \in \mathbb{F}$, the above inequality reduces to

$$
\sum_{i=1}^{p} u_{i}\left[f_{i}(x)-\lambda g_{i}(x)\right] \geq 0 .
$$

Now using (2.11) and Lemma 2.1, we see that

$$
\varphi(x)=\max _{a \in U} \frac{\sum_{i=1}^{p} a_{i} f_{i}(x)}{\sum_{i=1}^{p} a_{i} g_{i}(x)} \geq \frac{\sum_{i=1}^{p} u_{i} f_{i}(x)}{\sum_{i=1}^{p} u_{i} g_{i}(x)} \geq \lambda .
$$

(b): The proof is similar to that of part (a).

(c): Suppose to the contrary that $\varphi(x)<\lambda$. This implies that

$$
f_{i}(x)-\lambda g_{i}(x)<0, i \in \underline{p} .
$$

Since

$$
\begin{aligned}
\Phi(x, u, v, w, \lambda) & =\sum_{i=1}^{p} u_{i}\left[f_{i}(x)-\lambda g_{i}(x)\right]+\sum_{j \in J_{0}} u_{j} G_{j}(x)+\sum_{k \in K_{0}} w_{k} H_{k}(x) \\
& \left.\leq \sum_{i=1}^{p} u_{i}\left[f_{i}(x)-\lambda g_{i}(x)\right] \quad \text { (by the primal feasibility of } x\right) \\
& <0 \quad(\text { by }(2.12)) \\
& \leq \Phi(y, u, v, w, \lambda) \quad(\text { by }(2.3)),
\end{aligned}
$$

it follows from the properties of $\bar{\phi}_{i}$ that

$$
\bar{\phi}_{i}(\Phi(x, u, v, w, \lambda)-\Phi(y, u, v, w, \lambda))<0 .
$$

Applying (i), the above inequality implies that

$$
\begin{array}{r}
\mathcal{F}\left(x, y ; \beta(x, y)\left\{\sum_{i=1}^{p} u_{i}\left[\nabla f_{i}(y)-\lambda \nabla g_{i}(y)\right]+\sum_{j \in J_{0}} u_{j} \nabla G_{j}(y)+\sum_{k \in K_{0}} w_{k} \nabla H_{k}(y), \eta(x, y)\right\}\right) \\
+\frac{1}{2}\left\langle\zeta(x, y),\left[\sum_{i=1}^{p} u_{i}\left[\nabla^{2} f_{i}(y)-\lambda \nabla^{2} g_{i}(y)\right]+\sum_{j \in J_{0}} u_{j} \nabla^{2} G_{j}(y)+\sum_{k \in K_{0}} w_{k} \nabla^{2} H_{k}(y)\right] z\right\rangle \\
\leq-\bar{\rho}(x, y)\|\theta(x, y)\|^{m} .
\end{array}
$$


As seen in the proof of Theorem 2.1, our assumptions in (ii) lead to

$$
\begin{aligned}
\mathcal{F}\left(x, y ; \beta(x, y) \sum_{t=1}^{M}\left[\sum_{j \in J_{t}} u_{j} \nabla G_{j}(y)+\sum_{k \in K_{t}} w_{k} \nabla H_{k}(y)\right]\right) & +\frac{1}{2}\left\langle\zeta(x, y), \sum_{t=1}^{M}\left[\sum_{j \in J_{t}} u_{j} \nabla^{2} G_{j}(y)\right.\right. \\
& \left.\left.+\sum_{k \in K_{t}} w_{k} \nabla^{2} H_{k}(y)\right] z\right\rangle \leq-\sum_{t=1}^{M} \tilde{\rho}_{t}(x, y)\|\theta(x, y)\|^{m}
\end{aligned}
$$

which when combined with (2.7) and (2.8) results in

$$
\begin{array}{r}
\mathcal{F}\left(x, y ; \beta(x, y)\left\{\sum_{i=1}^{p} u_{i}\left[\nabla f_{i}(y)-\lambda \nabla g_{i}(y)\right]+\sum_{j \in J_{0}} u_{j} \nabla G_{j}(y)+\sum_{k \in K_{0}} w_{k} \nabla H_{k}(y), \eta(x, y)\right\}\right)+ \\
\frac{1}{2}\left\langle\zeta(x, y),\left\{\sum_{i=1}^{p} u_{i}\left[\nabla^{2} f_{i}(y)-\lambda \nabla^{2} g_{i}(y)\right]+\sum_{j \in J_{0}} u_{j} \nabla^{2} G_{j}(y)+\sum_{k \in K_{0}} w_{k} \nabla^{2} H_{k}(y)\right\} z\right\rangle \\
\geq \sum_{t=1}^{M} \tilde{\rho}_{t}(x, y)\|\theta(x, y)\|^{m} .
\end{array}
$$

In view of (iii), this inequality contradicts (2.13). Hence, $\varphi(x) \geq \lambda$.

(d): The proof is similar to that of part (c).

Theorem 2.2. (Strong Duality) Let $x^{*}$ be a normal optimal solution of $(P)$, let $\lambda^{*}=\varphi\left(x^{*}\right)$, and assume that any one of the four sets of conditions specified in Theorem 2.1 is satisfied for all feasible solutions of (DI). Then for each $z^{*} \in C\left(x^{*}\right)$, there exist $u^{*} \in U, v^{*} \in \mathbb{R}_{+}^{q}$, and $w^{*} \in \mathbb{R}^{r}$ such that $x^{*}$ is an optimal solution of $(D I)$ and $\varphi\left(x^{*}\right)=\lambda^{*}$.

Proof

Since $x^{*}$ is a normal optimal solution of $(P)$, by Theorem 2.1, for each $z^{*} \in C\left(x^{*}\right)$, there exist $u^{*}, v^{*}, w^{*}$, and $\lambda^{*}\left(=\varphi\left(x^{*}\right)\right)$, as specified above, such that $x^{*}$ is a feasible solution of $(D I)$. If, for each $z^{*} \in C\left(x^{*}\right)$, there exist $u^{*}, v^{*}, w^{*}$, and $\lambda^{*}\left(=\varphi\left(x^{*}\right)\right)$, as specified above, $x^{*}$ were not optimal, then there would exist a feasible solution $\tilde{x}$ such that $\tilde{\lambda}>\lambda^{*}=\varphi\left(x^{*}\right)$ which contradicts Theorem 2.1. Therefore, $x^{*}$ is an optimal solution of $(D I)$ with respect to $z^{*} \in C\left(x^{*}\right), u^{*}, v^{*}, w^{*}$, and $\lambda^{*}\left(=\varphi\left(x^{*}\right)\right)$.

Theorem 2.3. (Strict Converse Duality) Let $x^{*}$ be a normal optimal solution of $(P)$, and let $\tilde{x}$ be an optimal solution of (DI) with respect to $\tilde{z}, \tilde{u}, \tilde{v}, \tilde{w}, \tilde{\lambda}$. Furthermore assume that any one of the following four sets of conditions holds:

(a) The assumptions specified in part (a) of Theorem 2.1 are satisfied for the feasible solution $\tilde{x}$ of (DI), $\bar{\phi}(a)>0 \Rightarrow a>0$, and the function $\xi \rightarrow \Phi(\xi, \tilde{u}, \tilde{v}, \tilde{w}, \tilde{\lambda})$ is strictly $(\mathcal{F}, \beta, \bar{\phi}, \bar{\rho}, \zeta, \theta, m)$-pseudosounivex at $\tilde{x}$.

(b) The assumptions specified in part (b) of Theorem 2.1 are satisfied for the feasible solution $\tilde{x}$ of (DI), $\bar{\phi}(a)>0 \Rightarrow a>0$, and the function $\xi \rightarrow \Phi(\xi, \tilde{u}, \tilde{v}, \tilde{w}, \tilde{\lambda})$ is $(\mathcal{F}, \beta, \bar{\phi}, \bar{\rho}, \zeta, \theta, m)$-quasisounivex at $\tilde{x}$.

(c) The assumptions specified in part (c) of Theorem 2.1 are satisfied for the feasible solution $\tilde{x}$ of (DI), $\bar{\phi}(a)>0 \Rightarrow a>0$, and the function $\xi \rightarrow \Phi(\xi, \tilde{u}, \tilde{v}, \tilde{w}, \tilde{\lambda})$ is $(\mathcal{F}, \beta, \bar{\phi}, \bar{\rho}, \zeta, \theta, m)$-quasisounivex at $\tilde{x}$.

(d) The assumptions specified in part (d) of Theorem 2.1 are satisfied for the feasible solution $\tilde{x}$ of (DI), $\bar{\phi}(a)>0 \Rightarrow a>0$, and the function $\xi \rightarrow \Phi(\xi, \tilde{u}, \tilde{v}, \tilde{w}, \tilde{\lambda})$ is $(\mathcal{F}, \beta, \bar{\phi}, \bar{\rho}, \zeta, \theta, m)$-quasisounivex at $\tilde{x}$.

Then $\tilde{x}=x^{*}$ and $\varphi\left(x^{*}\right)=\tilde{\lambda}$.

Proof

Since $x^{*}$ is a normal optimal solution of $(P)$, by Theorem 2.1, there exist $\tilde{u}, \tilde{v}, \tilde{w}$, and $\tilde{\lambda}$ such that $\tilde{x}$ (with respect to $\tilde{z}, \tilde{u}, \tilde{v}, \tilde{w}, \tilde{\lambda})$ is a feasible solution of $(D I)$ and $\varphi\left(x^{*}\right)=\lambda^{*}$. 
(a): Suppose to the contrary that $\tilde{x} \neq x^{*}$. Now proceeding as in the proof of part (a) of Theorem 2.1 (with $x$ replaced by $x^{*}$ and $y$ by $\tilde{x}$, we arrive at the strict inequality

$$
\sum_{i=1}^{p} \tilde{u}_{i}\left[f_{i}(x)-\tilde{\lambda} g_{i}(x)\right]>0 .
$$

Using this inequality along with Lemma 2.1 , as in the proof of Theorem 2.1 , we get $\varphi\left(x^{*}\right)>\tilde{\lambda}$ which contradicts the fact that $\varphi\left(x^{*}\right)=\lambda^{*} \leq \tilde{\lambda}$. (b) - (d) : The proofs are similar to that of part (a).

In Theorems 2.1 - 2.3, various generalized $(\mathcal{F}, \beta, \phi, \rho, \zeta, \theta, m)$-sounivexity conditions were imposed on the function $\xi \rightarrow \Phi(\xi, u, v, w, \lambda)$, which is the weighted sum of the functions $\xi \rightarrow \Phi_{i}(\xi, v, w, \lambda), i \in p$. In the next few theorems, we shall assume that the individual functions $\xi \rightarrow \Phi_{i}(\xi, v, w, \lambda), i \in \underline{p}$, satisfy appropriate generalized $(\mathcal{F}, \beta, \phi, \rho, \zeta, \theta, m)$-sounivexity hypotheses.

Theorem 2.4. (Weak Duality) Let $x$ and $y$ be arbitrary feasible solutions of $(P)$ and (DI), respectively. Furthermore, assume that any one of the following seven sets of hypotheses is satisfied:

(a) (i) for each $i \in I_{+} \equiv\left\{i \in p: u_{i}>0\right\}, \xi \rightarrow \Phi_{i}(\xi, v, w, \lambda)$ is $\left(\mathcal{F}, \beta, \bar{\phi}_{i}, \bar{\rho}_{i}, \zeta, \theta, m\right)$-pseudosounivex at y, $\bar{\phi}_{i}$ is strictly increasing, and $\bar{\phi}_{i}(0)=0$;

(ii) for each $t \in \underline{M}, \xi \rightarrow \Lambda_{t}(\xi, v, w)$ is $\left(\mathcal{F}, \beta, \tilde{\phi}_{t}, \tilde{\rho}_{t}, \zeta, \theta, m\right)$-quasisounivex at $y, \tilde{\phi}_{t}$ is increasing, and $\tilde{\phi}_{t}(0)=0$

(iii) $\sum_{i \in I_{+}} u_{i} \bar{\rho}_{i}(x, y)+\sum_{t=1}^{M} \tilde{\rho}_{t}(x, y) \geq 0$;

(b) (i) for each $i \in I_{+}, \xi \rightarrow \Phi_{i}(\xi, v, w, \lambda)$ is prestrictly $\left(\mathcal{F}, \beta, \bar{\phi}_{i}, \bar{\rho}_{i}, \zeta, \theta, m\right)$-quasisounivex at $y, \bar{\phi}_{i}$ is strictly increasing, and $\bar{\phi}_{i}(0)=0$;

(ii) for each $t \in \underline{m}, \xi \rightarrow \Lambda_{t}(\xi, v, w)$ is strictly $\left(\mathcal{F}, \beta, \tilde{\phi}_{t}, \tilde{\rho}_{t}, \zeta, \theta, m\right)$-pseudosounivex at $y, \tilde{\phi}_{t}$ is increasing, and $\tilde{\phi}_{t}(0)=0$;

(iii) $\sum_{i \in I_{+}} u_{i} \bar{\rho}_{i}(x, y)+\sum_{t=1}^{M} \tilde{\rho}_{t}(x, y) \geq 0$;

(c) (i) for each $i \in I_{+}, \xi \rightarrow \Phi_{i}(\xi, v, w, \lambda)$ is prestrictly $\left(\mathcal{F}, \beta, \bar{\phi}_{i}, \bar{\rho}_{i}, \zeta, \theta, m\right)$-quasisounivex at $y$, $\bar{\phi}_{i}$ is strictly increasing, and $\bar{\phi}_{i}(0)=0$;

(ii) for each $t \in \underline{M}, \xi \rightarrow \Lambda_{t}(\xi, v, w)$ is $\left(\mathcal{F}, \beta, \tilde{\phi}_{t}, \tilde{\rho}_{t}, \zeta, \theta\right)$-quasisounivex at $y$, $\tilde{\phi}_{t}$ is increasing, and $\tilde{\phi}_{t}(0)=$ 0 ;

(iii) $\sum_{i \in I_{+}} u_{i} \bar{\rho}_{i}(x, y)+\sum_{t=1}^{M} \tilde{\rho}_{t}(x, y)>0$;

(d) (i) for each $i \in I_{1+}, \xi \rightarrow \Phi_{i}(\xi, v, w, \lambda)$ is $\left(\mathcal{F}, \beta, \bar{\phi}_{i}, \bar{\rho}_{i}, \zeta, \theta, m\right)$-pseudosounivex at $y$, for each $i \in$ $I_{2+}, \xi \rightarrow \Phi_{i}(\xi, v, w, \lambda)$ is prestrictly $\left(\mathcal{F}, \beta, \bar{\phi}_{i}, \bar{\rho}_{i}, \zeta, \theta, m\right)$-quasisounivex at $y$, and for each $i \in I_{+}, \bar{\phi}_{i}$ is strictly increasing and $\bar{\phi}_{i}(0)=0$, where $\left\{I_{1+}, I_{2+}\right\}$ is a partition of $I_{+}$;

(ii) for each $t \in \underline{M}, \xi \rightarrow \Lambda_{t}(\xi, v, w)$ is strictly $\left(\mathcal{F}, \beta, \tilde{\phi}_{t}, \tilde{\rho}_{t}, \zeta, \theta, m\right)$-pseudosounivex at $y, \tilde{\phi}_{t}$ is increasing, and $\tilde{\phi}_{t}(0)=0$;

(iii) $\sum_{i \in I_{+}} u_{i} \bar{\rho}_{i}(x, y)+\sum_{t=1}^{M} \tilde{\rho}_{t}(x, y) \geq 0$;

(e) (i) for each $i \in I_{1+} \neq \emptyset, \xi \rightarrow \Phi_{i}(\xi, v, w, \lambda)$ is $\left(\mathcal{F}, \beta, \bar{\phi}_{i}, \bar{\rho}_{i}, \zeta, \theta, m\right)$-pseudosounivex at $y$, for each $i \in$ $I_{2+}, \xi \rightarrow \Phi_{i}(\xi, v, w, \lambda)$ is prestrictly $\left(\mathcal{F}, \beta, \bar{\phi}_{i}, \bar{\rho}_{i}, \zeta, \theta\right)$-quasisounivex at $y$, and for each $i \in I_{+}, \bar{\phi}_{i}$ is strictly increasing and $\bar{\phi}_{i}(0)=0$, where $\left\{I_{1+}, I_{2+}\right\}$ is a partition of $I_{+}$;

(ii) for each $t \in \underline{M}, \xi \rightarrow \Lambda_{t}(\xi, v, w)$ is $\left(\mathcal{F}, \beta, \tilde{\phi}_{t}, \tilde{\rho}_{t}, \zeta, \theta, m\right)$-quasisounivex at $y, \tilde{\phi}_{t}$ is increasing, and $\tilde{\phi}_{t}(0)=0$

(iii) $\sum_{i \in I_{+}} u_{i} \bar{\rho}_{i}(x, y)+\sum_{t=1}^{M} \tilde{\rho}_{t}(x, y) \geq 0$;

(f) (i) for each $i \in I_{+}, \xi \rightarrow \Phi_{i}(\xi, v, \lambda, \bar{t}, \bar{s})$ is prestrictly $\left(\mathcal{F}, \beta, \bar{\phi}_{i}, \bar{\rho}_{i}, \zeta, \theta, m\right)$-quasisounivex at y, $\bar{\phi}_{i}$ is strictly increasing, and $\bar{\phi}_{i}(0)=0$; 
(ii) for each $t \in \underline{M_{1}} \neq \emptyset, \xi \rightarrow \Lambda_{t}(\xi, v, w)$ is strictly $\left(\mathcal{F}, \beta, \tilde{\phi}_{t}, \tilde{\rho}_{t}, \zeta, \theta, m\right)$-pseudosounivex at y, for each $t \in$ $\underline{M_{2}}, \xi \rightarrow \Lambda_{t} \overline{(\xi, v, w)}$ is $\left(\mathcal{F}, \beta, \tilde{\phi}_{t}, \tilde{\rho}_{t}, \zeta, \theta, m\right)$-quasisounivex at $y$, and for each $t \in \underline{M}, \tilde{\phi}_{t}$ is increasing and $\tilde{\phi}_{t}(0)=0$, where $\left\{\underline{M_{1}}, \underline{M_{2}}\right\}$ is a partition of $\underline{M}$;

(iii) $\sum_{i \in I_{+}} u_{i} \bar{\rho}_{i}(x, y)+\sum_{t=1}^{M} \tilde{\rho}_{t}(x, y) \geq 0$;

(g) (i) for each $i \in I_{1+}, \xi \rightarrow \Phi_{i}(\xi, v, w, \lambda)$ is $\left(\mathcal{F}, \beta, \bar{\phi}_{i}, \bar{\rho}_{i}, \zeta, \theta, m\right)$-pseudosounivex at $y$, for each $i \in$ $I_{2+}, \xi \rightarrow \Phi_{i}(\xi, v, w, \lambda)$ is prestrictly $\left(\mathcal{F}, \beta, \bar{\phi}_{i}, \bar{\rho}_{i}, \zeta, \theta, m\right)$-quasisounivex at $y$, and for each $i \in I_{+}, \bar{\phi}_{i}$ is strictly increasing and $\bar{\phi}_{i}(0)=0$, where $\left\{I_{1+}, I_{2+}\right\}$ is a partition of $I_{+}$;

(ii) for each $t \in \underline{M_{1}}, \xi \rightarrow \Lambda_{t}(\xi, v, w)$ is strictly $\left(\mathcal{F}, \beta, \tilde{\phi}_{t}, \tilde{\rho}_{t}, \zeta, \theta, m\right)$-pseudosounivex at $y$, for each $t \in$ $\underline{M_{2}}, \xi \rightarrow \Lambda_{t} \overline{(\xi, v, w)}$ is $\left(\mathcal{F}, \beta, \tilde{\phi}_{t}, \tilde{\rho}_{t}, \zeta, \theta, m\right)$-quasisounivex at $y$, and for $t \in \underline{M}, \tilde{\phi}_{t}$ is increasing and $\underline{\tilde{\phi}_{t}}(0)=0$, where $\left\{\underline{M_{1}}, \underline{M_{2}}\right\}$ is a partition of $\underline{M}$;

(iii) $\sum_{i \in I_{+}} u_{i} \bar{\rho}_{i}(x, y)+\sum_{t=1}^{M} \tilde{\rho}_{t}(x, y) \geq 0$;

(iv) $I_{1+} \neq \emptyset, \underline{M_{1}} \neq \emptyset$, or $\sum_{i \in I_{+}} u_{i} \bar{\rho}_{i}(x, y)+\sum_{t=1}^{M} \tilde{\rho}_{t}(x, y)>0$.

Then $\varphi(x) \geq \lambda$.

Proof

(a) : Suppose to the contrary that $\varphi(x)<\lambda$. This implies that

$$
f_{i}(x)-\lambda g_{i}(x)<0, i \in \underline{p} .
$$

Keeping in mind that $u \geq 0$, we see that for each $i \in I_{+}$,

$$
\begin{aligned}
\Phi_{i}(x, v, \lambda) & =f_{i}(x)-\lambda g_{i}(x)+\sum_{j \in J_{0}} u_{j} G_{j}(x)+\sum_{k \in K_{0}} w_{k} H_{k}(x) \\
& \left.\leq f_{i}(x)-\lambda g_{i}(x) \quad \text { (by the primal feasibility of } x\right) \\
& <0 \quad(\text { by }(2.14)) \\
& \leq \Phi_{i}(y, v, \lambda) \quad(\text { by }(2.3)),
\end{aligned}
$$

and so it follows from the properties of $\bar{\phi}_{i}$ that

$$
\bar{\phi}_{i}\left(\Phi_{i}(x, v, \lambda)-\Phi_{i}(y, v, \lambda)\right)<0 .
$$

Hence, by (i), for each $i \in I_{+}$, the above inequality implies that

$$
\begin{aligned}
& \mathcal{F}(x, y ; \beta(x, y)\{\left.\left\{f_{i}(y)-\lambda \nabla g_{i}(y)+\sum_{j \in J_{0}} u_{j} \nabla G_{j}(y)+\sum_{k \in K_{0}} w_{k} \nabla H_{k}(y)\right\}\right) \\
&+ \frac{1}{2}\left\langle\zeta(x, y),\left[\nabla^{2} f_{i}(y)-\lambda \nabla^{2} g_{i}(y)+\sum_{j \in J_{0}} u_{j} \nabla^{2} G_{j}(y)+\sum_{k \in K_{0}} w_{k} \nabla^{2} H_{k}(y)\right] z\right\rangle \\
&<-\bar{\rho}_{i}(x, y)\|\theta(x, y)\|^{m} .
\end{aligned}
$$

Since $u \geq 0, u_{i}=0$ for each $i \in \underline{p} \backslash I_{+}, \sum_{i=1}^{p} u_{i}=1$, and $\mathcal{F}(x, y ; \cdot)$ is sublinear, the above inequalities yield

$$
\begin{array}{r}
\mathcal{F}\left(x, y ; \beta(x, y)\left\{\sum_{i=1}^{p} u_{i}\left[\nabla f_{i}(y)-\lambda \nabla g_{i}(y)\right]+\sum_{j \in J_{0}} u_{j} \nabla G_{j}(y)+\sum_{k \in K_{0}} w_{k} \nabla H_{k}(y), \eta(x, y)\right\}\right)+ \\
\frac{1}{2}\left\langle\zeta(x, y),\left\{\sum_{i=1}^{p} u_{i}\left[\nabla^{2} f_{i}(y)-\lambda \nabla^{2} g_{i}(y)\right]+\sum_{j \in J_{0}} u_{j} \nabla^{2} G_{j}(y)+\sum_{k \in K_{0}} w_{k} \nabla^{2} H_{k}\left(x^{*}\right)\right\} z\right\rangle \\
<-\sum_{i \in I_{+}} u_{i} \bar{\rho}_{i}(x, y)\|\theta(x, y)\|^{m} .
\end{array}
$$


As seen in the proof of Theorem 2.1, our assumptions in (ii) lead to

$$
\begin{aligned}
\mathcal{F}\left(x, y ; \beta(x, y) \sum_{t=1}^{M}\left[\sum_{j \in J_{t}} u_{j} \nabla G_{j}(y)+\sum_{k \in K_{t}} w_{k} \nabla H_{k}(y)\right]\right) & +\frac{1}{2}\left\langle\zeta(x, y), \sum_{t=1}^{M}\left[\sum_{j \in J_{t}} u_{j} \nabla^{2} G_{j}(y)\right.\right. \\
& \left.\left.+\sum_{k \in K_{t}} w_{k} \nabla^{2} H_{k}(y)\right] z\right\rangle \leq-\sum_{t=1}^{M} \tilde{\rho}_{t}(x, y)\|\theta(x, y)\|^{m},
\end{aligned}
$$

which when combined with (2.7) and (2.8) results in

$$
\begin{gathered}
\mathcal{F}\left(x, y ; \beta(x, y)\left\{\sum_{i=1}^{p} u_{i}\left[\nabla f_{i}(y)-\lambda \nabla g_{i}(y)\right]+\sum_{j \in J_{0}} u_{j} \nabla G_{j}(y)+\sum_{k \in K_{0}} w_{k} \nabla H_{k}(, y), \eta(x, y)\right\}\right)+ \\
\frac{1}{2}\left\langle\zeta(x, y),\left\{\sum_{i=1}^{p} u_{i}\left[\nabla^{2} f_{i}(y)-\lambda \nabla^{2} g_{i}(y)\right]+\sum_{j \in J_{0}} u_{j} \nabla^{2} G_{j}(y)\right.\right. \\
\left.\left.+\sum_{k \in K_{0}} w_{k} \nabla^{2} H_{k}(y)\right\} z\right\rangle \geq \sum_{t=1}^{M} \tilde{\rho}_{t}(x, y)\|\theta(x, y)\|^{m} .
\end{gathered}
$$

In view of (iii), this inequality contradicts (2.15). Hence, $\varphi(x) \geq \lambda$.

(b) - (g) : The proofs are similar to that of part (a).

Theorem 2.5. (Strong Duality) Let $x^{*}$ be a normal optimal solution of $(P)$ and assume that any one of the seven sets of conditions set forth in Theorem 2.4 is satisfied for all feasible solutions of $(D I)$. Then for each $z^{*} \in C\left(x^{*}\right)$, there exist $u^{*}, v^{*}, w^{*}$, and $\lambda^{*}$ such that $x^{*}$ is an optimal solution of $(D I)$ and $\varphi\left(x^{*}\right)=\lambda^{*}$.

Proof

The proof is similar to that of Theorem 2.2.

\section{Duality Model II}

In this section we discuss two additional duality models for $(P)$. In these duality formulations, we utilize a partition of $\underline{p}$ in addition to those of $\underline{q}$ and $\underline{r}$. This partitioning scheme, which is a slightly extended version of the one initially proposed by Mond and Weir [6], was used by Yang [13] for formulating a generalized duality model for a multiobjective fractional programming problem. In our duality theorems, we impose appropriate generalized $(\mathcal{F}, \beta, \phi, \rho, \zeta, \theta, m)$-sounivexity requirements on certain combinations of the problem functions.

Let $\left\{I_{0}, I_{1}, \ldots, I_{\ell}\right\}$ be a partition of $p$ such that $\mathcal{L}=\{0,1,2, \ldots, \ell\} \subset \mathcal{M}=\{0,1, \ldots, M\}$, and let the real-

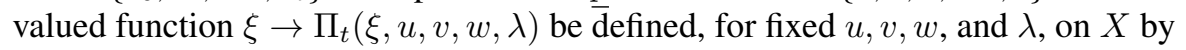

$$
\Pi_{t}(\xi, u, v, w, \lambda)=\sum_{i \in I_{t}} u_{i}\left[f_{i}(x)-\lambda g_{i}(x)\right]+\sum_{j \in J_{t}} v_{j} G_{j}(x)+\sum_{k \in K_{t}} w_{k} H_{k}(x), \quad t \in \mathcal{M} .
$$

Consider the following two problems:

$(D I I) \quad$ Maximize $\lambda$

subject to

$$
\sum_{i=1}^{p} u_{i}\left[\nabla f_{i}(y)-\lambda \nabla g_{i}(y)\right]+\sum_{j=1}^{q} v_{j} \nabla G_{j}(y)+\sum_{k=1}^{r} w_{k} \nabla H_{k}(y)=0,
$$




$$
\begin{gathered}
\left\langle z,\left\{\sum_{i=1}^{p} u_{i}\left[\nabla^{2} f_{i}(y)-\lambda \nabla^{2} g_{i}(y)\right]+\sum_{j=1}^{q} v_{j} \nabla^{2} G_{j}(y)+\sum_{k=1}^{r} w_{k} \nabla^{2} H_{k}(y)\right\} z\right\rangle \geq 0 \\
\sum_{t \in I_{t}} u_{i}\left[f_{i}(y)-\lambda g_{i}(y)\right]+\sum_{j \in J_{t}} v_{j} G_{j}(y)+\sum_{k \in K_{t}} w_{k} H_{k}(y) \geq 0, \quad t \in \mathcal{M}, \\
\sum_{j \in J_{t}} v_{j} G_{j}(y)+\sum_{k \in K_{t}} w_{k} H_{k}(y) \geq 0, \quad t \in \mathcal{L} \backslash M \\
y \in X, z \in C(y), u \in \mathbb{R}^{p}, u \geq 0, \sum_{i=1}^{p} u_{i}=1, v \in \mathbb{R}_{+}^{q}, w \in \mathbb{R}^{r}, \lambda \in \mathbb{R}_{+}
\end{gathered}
$$

$(\tilde{D} I I) \quad$ Maximize $\quad \lambda$

subject to (3.2) - (3.5) and

$$
\mathcal{F}\left(x, y ; \sum_{i=1}^{p} u_{i}\left[\nabla f_{i}(y)-\lambda \nabla g_{i}(y)\right]+\sum_{j=1}^{q} v_{j} \nabla G_{j}(y)+\sum_{k=1}^{r} w_{k} \nabla H_{k}(y)\right) \geq 0 \text { for all } x \in \mathbb{F},
$$

where $\mathcal{F}(x, y ; \cdot)$ is a sublinear function from $\mathbb{R}^{n}$ to $\mathbb{R}$.

The feasible set $\mathbb{F}_{(D I I)}$ (assumed to be nonempty) of $(D I I)$ is defined as:

$$
\mathbb{F}_{(D I I)}=\left\{y \in X: z \in \mathbb{R}^{n} ; u \in \mathbb{R}^{p}, u \geq 0, \sum_{i=1}^{p} u_{i}=1 ; v \in \mathbb{R}_{+}^{q}, v_{i} \geq 0 ; w \in \mathbb{R}^{r} ; \lambda \in \mathbb{R}_{+}\right\}
$$

The comments and observations made earlier about the relationship between $(D I)$ and $(\tilde{D} I)$ are, of course, also valid for $(D I I)$ and $(\tilde{D} I I)$.

The following two theorems show that $(D I I)$ is a dual problem for $(P)$.

Theorem 3.1. (Weak Duality) Let $x$ and $y$ be arbitrary feasible solutions of $(P)$ and (DII), respectively. Furthermore, assume that any one of the following seven sets of hypotheses is satisfied:

(a) (i) for each $t \in \mathcal{L}, \xi \rightarrow \Pi_{t}(\xi, u, v, w, \lambda)$ is strictly $\left(\mathcal{F}, \beta, \phi_{t}, \rho_{t}, \zeta, \theta, m\right)$-pseudosounivex at y, $\phi_{t}$ is increasing, and $\phi_{t}(0)=0$;

(ii) for each $t \in \mathcal{M} \backslash \mathcal{L}, \xi \rightarrow \Lambda_{t}(\xi, v, w)$ is $\left(\mathcal{F}, \beta, \phi_{t}, \rho_{t}, \zeta, \theta, m\right)$-quasisounivex at $y$, $\phi_{t}$ is increasing, and $\phi_{t}(0)=0$

(iii) $\sum_{t \in \mathcal{M}} \rho_{t}(x, y) \geq 0$ for all $x \in \mathbb{F}$;

(b) (i) for each $t \in \mathcal{L}, \xi \rightarrow \Pi_{t}(\xi, u, v, w, \lambda)$ is prestrictly $\left(\mathcal{F}, \beta, \phi_{t}, \rho_{t}, \zeta, \theta, m\right)$-quasisounivex at y, $\phi_{t}$ is increasing, and $\phi_{t}(0)=0$;

(ii) for each $t \in \mathcal{M} \backslash \mathcal{L}, \xi \rightarrow \Lambda_{t}(\xi, v, w)$ is strictly $\left(\mathcal{F}, \beta, \phi_{t}, \rho_{t}, \zeta, \theta, m\right)$-pseudosounivex at y, $\phi_{t}$ is increasing, and $\phi_{t}(0)=0$;

(iii) $\sum_{t \in \mathcal{M}} \rho_{t}(x, y) \geq 0$ for all $x \in \mathbb{F}$;

(c) (i) for each $t \in \mathcal{L}, \xi \rightarrow \Pi_{t}(\xi, u, v, w, \lambda)$ is prestrictly $\left(\mathcal{F}, \beta, \phi_{t}, \rho_{t}, \zeta, \theta, m\right)$-quasisounivex at y, $\phi_{t}$ is increasing, and $\phi_{t}(0)=0$;

(ii) for each $t \in \mathcal{M} \backslash \mathcal{L}, \xi \rightarrow \Lambda_{t}(\xi, v, w)$ is $\left(\mathcal{F}, \beta, \phi_{t}, \rho_{t}, \zeta, \theta, m\right)$-quasisounivex at $y$, $\phi_{t}$ is increasing, and $\phi_{t}(0)=0$

(iii) $\sum_{t \in \mathcal{M}} \rho_{t}(x, y)>0$ for all $x \in \mathbb{F}$; 
(d) (i) for each $t \in \mathcal{L}_{1}, \xi \rightarrow \Pi_{t}(\xi, u, v, w, \lambda)$ is strictly $\left(\mathcal{F}, \beta, \phi_{t}, \rho_{t}, \theta, m\right)$-pseudosounivex at y, for each $t \in$ $\mathcal{L}_{2}, \xi \rightarrow \Pi_{t}(\xi, u, v, w, \lambda)$ is prestrictly $\left(\mathcal{F}, \beta, \phi_{t}, \rho_{t}, \zeta, \theta, m\right)$-quasisounivex at $y$, and for each $t \in \mathcal{L}, \phi_{t}$ is increasing and $\phi_{t}(0)=0$, where $\left\{\mathcal{L}_{1}, \mathcal{L}_{2}\right\}$ is a partition of $\mathcal{L}$;

(ii) for each $t \in \mathcal{M} \backslash \mathcal{L}, \xi \rightarrow \Lambda_{t}(\xi, v, w)$ is strictly $\left(\mathcal{F}, \beta, \phi_{t}, \rho_{t}, \zeta, \theta, m\right)$-pseudosounivex at $y$, $\phi_{t}$ is increasing, and $\phi_{t}(0)=0$;

(iii) $\sum_{t \in \mathcal{M}} \rho_{t}(x, y) \geq 0$ for all $x \in \mathbb{F}$;

(e) (i) for each $t \in \mathcal{L}_{1} \neq \emptyset, \xi \rightarrow \Pi_{t}(\xi, u, v, w, \lambda)$ is strictly $\left(\mathcal{F}, \beta, \phi_{t}, \rho_{t}, \zeta, \theta, m\right)$-pseudosounivex at y, for each $t \in \mathcal{L}_{2}, \xi \rightarrow \Pi_{t}(\xi, u, v, w, \lambda)$ is prestrictly $\left(\mathcal{F}, \beta, \phi_{t}, \rho_{t}, \zeta, \theta, m\right)$-quasisounivex at $y$, and for each $t \in \mathcal{L}, \phi_{t}$ is increasing and $\phi_{t}(0)=0$, where $\left\{\mathcal{L}_{1}, \mathcal{L}_{2}\right\}$ is a partition of $\mathcal{L}$;

(ii) for each $t \in \mathcal{M} \backslash \mathcal{L}, \xi \rightarrow \Lambda_{t}(\xi, v, w)$ is $\left(\mathcal{F}, \beta, \phi_{t}, \rho_{t}, \zeta, \theta, m\right)$-quasisounivex at $y$, $\phi_{t}$ is increasing, and $\phi_{t}(0)=0$

(iii) $\sum_{t \in \mathcal{M}} \rho_{t}(x, y) \geq 0$ for all $x \in \mathbb{F}$;

(f) (i) for each $t \in \mathcal{L}, \xi \rightarrow \Pi_{t}(\xi, u, v, w, \lambda)$ is prestrictly $\left(\mathcal{F}, \beta, \phi_{t}, \rho_{t}, \zeta, \theta, m\right)$-quasisounivex at y, $\phi_{t}$ is increasing, and $\phi_{t}(0)=0$;

(ii) for each $t \in(\mathcal{M} \backslash \mathcal{L})_{1} \neq \emptyset, \xi \rightarrow \Lambda_{t}(\xi, v, w)$ is strictly $\left(\mathcal{F}, \beta, \phi_{t}, \rho_{t}, \zeta, \theta, m\right)$-pseudosounivex at $y$, for each $t \in(\mathcal{M} \backslash \mathcal{L})_{2}, \xi \rightarrow \Lambda_{t}(\xi, v, w)$ is $\left(\mathcal{F}, \beta, \phi_{t}, \rho_{t}, \zeta, \theta, m\right)$-quasisounivex at $y$, and for each $t \in \mathcal{L}, \phi_{t}$ is increasing and $\phi_{t}(0)=0$, where $\left\{(\mathcal{M} \backslash \mathcal{L})_{1},(\mathcal{M} \backslash \mathcal{L})_{2}\right\}$ is a partition of $\mathcal{M} \backslash \mathcal{L}$;

(iii) $\sum_{t \in \mathcal{M}} \rho_{t}(x, y) \geq 0$ for all $x \in \mathbb{F}$;

(g) (i) for each $t \in \mathcal{L}_{1}, \xi \rightarrow \Pi_{t}(\xi, u, v, w, \lambda)$ is $\left(\mathcal{F}, \beta, \phi_{t}, \rho_{t}, \zeta, \theta, m\right)$-pseudosounivex at y, for each $t \in$ $\mathcal{L}_{2}, \xi \rightarrow \Pi_{t}(\xi, u, v, w, \lambda)$ is prestrictly $\left(\mathcal{F}, \beta, \phi_{t}, \rho_{t}, \zeta, \theta, m\right)$-quasisounivex at $y$, and for each $t \in \mathcal{L}, \phi_{t}$ is increasing and $\phi_{t}(0)=0$, where $\left\{\mathcal{L}_{1}, \mathcal{L}_{2}\right\}$ is a partition of $\mathcal{L}$;

(ii) for each $t \in(\mathcal{M} \backslash \mathcal{L})_{1}, \xi \rightarrow \Lambda_{t}(z, v, w)$ is strictly $\left(\mathcal{F}, \beta, \phi_{t}, \rho_{t}, \zeta, \theta, m\right)$-pseudosounivex at y, for each $t \in(\mathcal{M} \backslash \mathcal{L})_{2}, \xi \rightarrow \Lambda_{t}(\xi, v, w)$ is $\left(\mathcal{F}, \beta, \phi_{t}, \rho_{t}, \zeta, \theta, m\right)$-quasisounivex at $y$, and for each $t \in \mathcal{M} \backslash \mathcal{L}, \phi_{t}$ is increasing and $\phi_{t}(0)=0$, where $\left\{(\mathcal{M} \backslash \mathcal{L})_{1},(\mathcal{M} \backslash \mathcal{L})_{2}\right\}$ is a partition of $\mathcal{M} \backslash \mathcal{L}$;

(iii) $\sum_{t \in \mathcal{M}} \rho_{t}(x, y) \geq 0$ for all $x \in \mathbb{F}$;

(iv) $\mathcal{L}_{1} \neq \emptyset,(\mathcal{M} \backslash \mathcal{L})_{1} \neq \emptyset$, or $\sum_{t \in \mathcal{M}} \rho_{t}(x, y)>0$.

Then $\varphi(x) \geq \lambda$.

Proof

(a) : Suppose to the contrary that $\varphi(x)<\lambda$. This implies that

$$
f_{i}(x)-\lambda g_{i}(x)<0, \quad i \in \underline{p} .
$$

Since $u \geq 0$ and $u \neq 0$, we see that for each $t \in \mathcal{L}$,

$$
\sum_{i \in I_{t}} u_{i}\left[f_{i}(x)-\lambda g_{i}(x)\right] \leq 0 .
$$

Now using this inequality, we see that for each $t \in \mathcal{L}$,

$$
\begin{aligned}
\Pi_{t}(x, u, v, w, \lambda)= & \sum_{i \in I_{t}} u_{i}\left[f_{i}(x)-\lambda g_{i}(x)\right]+\sum_{j \in J_{t}} v_{j} G_{j}(x)+\sum_{k \in K_{t}} w_{k} H_{k}(x) \\
\leq & \sum_{i \in I_{t}} u_{i}\left[f_{i}(x)-\lambda g_{i}(x)\right] \quad \text { (by the primal feasibility of } x \text { ) } \\
\leq & 0 \quad(\text { by }(3.6)) \\
\leq & \sum_{i \in I_{t}} u_{i}\left[f_{i}(y)-\lambda g_{i}(y)\right]+\sum_{j \in J_{t}} v_{j} G_{j}(y)+\sum_{k \in K_{t}} w_{k} H_{k}(y) \\
& (\text { by }(3.3)) \\
& =\Pi_{t}(y, u, v, w, \lambda),
\end{aligned}
$$


and hence

$$
\phi_{t}\left(\Pi_{t}(x, u, v, w, \lambda)-\Pi_{t}(y, u, v, w, \lambda)\right) \leq 0 .
$$

Hence, by (i), for each $t \in \mathcal{L}$, the above inequality implies that

$$
\begin{aligned}
\mathcal{F}\left(x, y ; \beta(x, y)\left\{\sum_{i \in I_{t}} u_{i}\left[\nabla f_{i}(y)-\lambda \nabla g_{i}(y)\right]+\sum_{j \in J_{t}} v_{j} \nabla G_{j}(y)\right.\right. & \left.\left.+\sum_{k \in K_{t}} w_{k} \nabla H_{k}(y)\right\}\right) \\
+\frac{1}{2}\left\langle\zeta(x, y),\left\{\sum_{i \in I_{t}} u_{i}\left[\nabla^{2} f_{i}(y)-\lambda \nabla^{2} g_{i}(y)\right]+\sum_{j \in J_{t}} v_{j} \nabla^{2} G_{j}(y)\right.\right. & \left.\left.+\sum_{k \in K_{t}} w_{k} \nabla^{2} H_{k}(y)\right\} z\right\rangle<-\rho_{t}(x, y)\|\theta(x, y)\|^{m} .
\end{aligned}
$$

Summing over $t \in \mathcal{L}$ and using the sublinearity of $\mathcal{F}(x, y ; \cdot)$, we obtain

$$
\begin{aligned}
\mathcal{F}\left(x, y ; \beta(x, y)\left\{\sum_{i=1}^{p} u_{i}\left[\nabla f_{i}(y)-\lambda \nabla g_{i}(y)\right]+\sum_{t \in \mathcal{L}}\left[\sum_{j \in J_{t}} v_{j} \nabla G_{j}(y)+\sum_{k \in K_{t}} w_{k} \nabla H_{k}(y)\right]\right\}\right) \\
+\frac{1}{2}\left\langle\zeta(x, y), \sum_{t \in \mathcal{L}}\left\{\sum_{i \in I_{t}} u_{i}\left[\nabla^{2} f_{i}(y)-\lambda \nabla^{2} g_{i}(y)\right]+\sum_{j \in J_{t}} v_{j} \nabla^{2} G_{j}(y)\right.\right. \\
\left.\left.+\sum_{k \in K_{t}} w_{k} \nabla^{2} H_{k}(y)\right\} z\right\rangle<-\sum_{t \in \mathcal{L}} \rho_{t}(x, y)\|\theta(x, y)\|^{m} .
\end{aligned}
$$

Proceeding as in the proof of Theorem 2.1, we find that for each $t \in \mathcal{M} \backslash \mathcal{L}$,

$$
\Lambda_{t}(x, v, w) \leq \Lambda_{t}(y, v, w)
$$

and so

which in view of (ii) implies that

$$
\phi_{t}\left(\Lambda_{t}(x, v, w)-\Lambda_{t}(y, v, w)\right) \leq 0
$$

$$
\begin{aligned}
\mathcal{F}\left(x, y ; \beta(x, y)\left[\sum_{j \in J_{t}} v_{j} \nabla G_{j}(y)+\sum_{k \in K_{t}} w_{k} \nabla H_{k}(y)\right]\right)+\frac{1}{2}\left\langle\zeta(x, y),\left[\sum_{j \in J_{t}} v_{j} \nabla^{2} G_{j}(y)\right.\right. & \\
& \left.\left.+\sum_{k \in K_{t}} w_{k} \nabla^{2} H_{k}(y)\right] z\right\rangle \leq-\rho_{t}(x, y)\|\theta(x, y)\|^{m} .
\end{aligned}
$$

Summing over $t \in \mathcal{M} \backslash \mathcal{L}$ and using the sublinearity of $\mathcal{F}(x, y ; \cdot)$, we get

$$
\begin{aligned}
\mathcal{F}\left(x, y ; \beta(x, y) \sum_{t \in \mathcal{M} \backslash \mathcal{L}}\left[\sum_{j \in J_{t}} v_{j} \nabla G_{j}(y)+\sum_{k \in K_{t}} w_{k} \nabla H_{k}(y)\right]\right)+\frac{1}{2}\left\langle\zeta(x, y), \sum_{t \in \mathcal{M} \backslash \mathcal{L}}\left[\sum_{j \in J_{t}} v_{j} \nabla^{2} G_{j}(y)\right.\right. \\
\left.\left.+\sum_{k \in K_{t}} w_{k} \nabla^{2} H_{k}(y)\right] z\right\rangle \leq-\sum_{t \in \mathcal{M} \backslash \mathcal{L}} \rho_{t}(x, y)\|\theta(x, y)\|^{m} .
\end{aligned}
$$

Now combining (3.7) and (3.8) and using (iii), we obtain

$$
\begin{aligned}
\mathcal{F}\left(x, y ; \beta(x, y)\left\{\sum_{i=1}^{p} u_{i}\left[\nabla f_{i}(y)-\lambda \nabla g_{i}(y)\right]\right.\right. & \left.\left.+\sum_{j=1}^{q} v_{j} \nabla G_{j}(y)+\sum_{k=1}^{r} w_{k} \nabla H_{k}(y)\right\}\right) \\
+\frac{1}{2}\left\langle\zeta(x, y),\left\{\sum_{i=1}^{p} u_{i}\left[\nabla^{2} f_{i}(y)-\lambda \nabla^{2} g_{i}(y)\right]+\sum_{j=1}^{q} v_{j} \nabla^{2} G_{j}(y)\right.\right. & \left.\left.+\sum_{k=1}^{r} w_{k} \nabla^{2} H_{k}(y)\right\} z\right\rangle<-\sum_{t \in \mathcal{M}} \rho_{t}(x, y)\|\theta(x, y)\|^{m} \leq 0 .
\end{aligned}
$$


Now multiplying (3.1) by $\beta(x, y)$, applying the sublinear function $\mathcal{F}(x, y ; \cdot)$ to both sides of the resulting equation, and then adding the equation to (3.2), we get

$$
\begin{aligned}
\mathcal{F}(x, y ; \beta(x, y)\{ & \left.\left.\sum_{i=1}^{p} u_{i}\left[\nabla f_{i}(y)-\lambda \nabla g_{i}(y)\right]+\sum_{j=1}^{q} v_{j} \nabla G_{j}(y)+\sum_{k=1}^{r} w_{k} \nabla H_{k}(y)\right\}\right) \\
& +\frac{1}{2}\left\langle\zeta(x, y),\left\{\sum_{i=1}^{p} u_{i}\left[\nabla^{2} f_{i}(y)-\lambda \nabla^{2} g_{i}(y)\right]+\sum_{j=1}^{q} v_{j} \nabla^{2} G_{j}(y)+\sum_{k=1}^{r} w_{k} \nabla^{2} H_{k}(y)\right\} z\right\rangle \geq 0,
\end{aligned}
$$

which contradicts (3.9). Therefore, we conclude that $\varphi(x) \geq \lambda$.

(b) - (g) : The proofs are similar to that of part (a).

Theorem 3.2. (Strong Duality) Let $x^{*}$ be a normal optimal solution of $(P)$ and assume that any one of the seven sets of conditions set forth in Theorem 3.1 is satisfied for all feasible solutions of (DII). Then for each $z^{*} \in C\left(x^{*}\right)$, there exist $u^{*}, v^{*}, w^{*}$, and $\lambda^{*}$ such that $x^{*}$ is an optimal solution of $(D I I)$ and $\varphi\left(x^{*}\right)=\lambda^{*}$.

Proof

The proof is similar to that of Theorem 2.2.

\section{Concluding Remarks}

It seems that the results presented in this paper will prove useful in investigating other related classes on nonlinear programming problems and applying similar generalized convexity concepts to nonlinear fractional programming problems, including finite and semiinfinite aspects, for example, a class of semiinfinite minmax fractional programming problems.

\section{REFERENCES}

1. W. Dinkelbach, On nonlinear fractional programming, Management Sci. 13 (1967), 492 - 498.

2. S. K. Gupta, D. Dangar and S. Kumar, Second-order duality for a nondifferentiable minmax fractional programming under generalized $\alpha$-univexity, Journal of Inequalities and Applications 2012 (2012): 187

3. M. A Hanson, Second order invexity and duality in mathematical programming, Opsearch 30 (1993), $313-320$.

4. Q. Hua, Y. Chena and J. Jianb, Second order duality for non-differentiable minmax fractional programming, International Journal of Computer Mathematics 89 (1) (2012), 11 - 16.

5. Z. Husain, I. Ahmad and S. Sharma, Second order duality for minmax fractional programming, Optimization Letters 3 (2009), 277 286.

6. B. Mond and T. Weir, Generalized concavity and duality, Generalized Concavity in Optimization and Economics (S. Schaible and W. T. Ziemba, eds.), Academic Press, New York, 1981, pp. 263 - 279.

7. S. Sharma and T. R. Gulati, Second order duality in minmax fractional programming with generalized univexity, Journal of Global Optimization 52 (2012), 161 - 169.

8. A. K. Tripathi, Second Order Duality in Multiobjective Fractional Programming with Square Root Term Under Generalized Univex Function, International Scholarly Research Notes 2014; 2014:541524.

9. R. U. Verma and G. J. Zalmai, Generalized second order parametric optimality conditions in semiinfinite discrete minmax fractional programming and second order $(\mathcal{F}, \beta, \phi, \rho, \theta, m$-univexity, Statistics, Optimization, \& Information Computing 4 (2016), 15 - 29.

10. R. U. Verma and G. J. Zalmai, Second-order parametric optimality conditions in discrete minmax fractional programming, Communications on Applied Nonlinear Analysis 23 (3) (2016), 1 - 32.

11. R. U. Verma, Hybrid parametric duality models in discrete minmax fractional programming problems on second-order optimality conditions, Transactions on Mathematical programming and Applications 5 (1) (2017), 89 - 120

12. R. U. Verma and G. J. Zalmai, Generalized second-order parameter-free optimality conditions in discrete minmax fractional programming, Communications on Applied Nonlinear Analysis 22 (2) (2015), 57 - 78.

13. X. Yang, Generalized convex duality for multiobjective fractional programs, Opsearch 31 (1994), 155 - 163.

14. G. J. Zalmai, Optimality conditions and duality for constrained measurable subset selection problems with minmax objective functions, Optimization 20 (1989), 377 - 395.

15. G. J. Zalmai, Generalized second-order $(F, \beta, \phi, \rho, \theta)$-univex functions and parametric duality models in semi infinite discrete minmax fractional programming, Advances in Nonlinear Variational Inequalities 15 (2) (2012), 63 - 91.

16. G. J. Zalmai, Hanson-Antczak-type generalized $(\alpha, \beta, \gamma, \xi, \eta, \rho, \theta)$-V-invex functions in semiinfinite minmax fractional programming, Part III: Second-order parametric duality models, Advances in Nonlinear Variational Inequalities 16 (2) (2013), 91 - 126. 